\title{
Large-system analysis of a CDMA dynamic channel under a Markovian input process
}

\author{
Ezio Biglieri*, Emanuele Grossi ${ }^{\dagger}$, Marco Lops ${ }^{\dagger}$, and Adrià Tauste Campo* \\ *Universitat Pompeu Fabra \\ Email: e.biglieri@ieee.org \\ ${ }^{\dagger}$ Universita di Cassino \\ Email: e.grossi@unicas.it \\ †Universita di Cassino \\ Email: lops@unicas.it \\ *Universitat Pompeu Fabra \\ Email: adria.tauste@upf.edu
}

\begin{abstract}
We study the minimum mean square error (MMSE) and the multiuser efficiency $\eta$ of large dynamic multiple access communication systems in which optimal multiuser detection is performed at the receiver as the number and the identities of active users is allowed to change at each transmission time. The system dynamics are ruled by a Markov model describing the evolution of the channel occupancy and a large-system analysis is performed when the number of observations grow large. Starting on the equivalent scalar channel and the fixed-point equation tying multiuser efficiency and MMSE, we extend it to the case of a dynamic channel, and derive lower and upper bounds for the MMSE (and, thus, for $\eta$ as well) holding true in the limit of large signal-to-noise ratios and increasingly large observation time $T$.
\end{abstract}

\section{INTRODUCTION}

In mobile multiple access communications, the number of active users, their location and other channel state parameters are variable with time. The estimate of these parameters has several applications to communication systems, e.g., user localization in wireless networks, neighbor discovery in ad hoc networks, and power-control strategy optimizations.

The classical approach to multiuser detection is based on the assumption that the number of active users is constant and known at the receiver, and corresponds to the maximum number of users entitled to access the system. However, this model is quite pessimistic in a dynamic environment where there is a considerable number of users that may remain inactive at any given time: for this reason, in [1] a more realistic framework has been outlined and a new class of detectors has been introduced. In particular, the problem of jointly estimating the number, the identities and the data of active users has been considered, while [2] assumes that also a number of continuous channel parameters are unknown at the receiver end.

The object of interest here is the large-system analysis of code division multiple access (CDMA) systems according to the dynamic environment given in [1]. Thus, this paper is aimed at analyzing the performance of a CDMA system in terms of multiuser efficiency when its natural dimensions (number of users, $K$, and spreading gain, $N$ ) tend to infinity keeping fixed their ratio $(\beta=K / N)$. The analysis will be based on recent tools developed from statistical physics. Of particular interest is the large-system equivalent single-user channel that is proved in [3] for a randomly spread CDMA system using the replica method analysis. Previous results on this topic are contained in [4], where the replica method has been applied to the static channel model described in [1] and the large system performance for joint data detection and user identification is given for a single instant time. The results are an extension of those corresponding to known users and allow evaluating the degradation due to the prior uncertainty as to the users' activity.

In this paper we address the large system analysis of a multiuser detector as identities of the active user is allowed to vary with time according to a known dynamic model. Minimum mean square error (MMSE) and multiuser efficiency are derived in this new scenario and an asymptotic analysis (as the observation time becomes increasingly large) is given, along with bounds on the MMSE and $\eta$. The rest of the paper is organized as follows. Next section contains the system model, and in particular the scalar channel model applicable in the large system limit. Section III presents the asymptotic (i.e., for large signal-to-noise ratio and large observation time) analysis, while Section IV is devoted to the validation of the analytical results through numerical simulations. Concluding remarks form the object of Section V.

\section{SySTEM MODEL}

Consider a synchronous BPSK-CDMA system with spreading factor $N$ and denote $K<N$ the maximum number of active users [3]. The signature of the $k-$ th user is modeled as a binary random sequence, $\mathbf{S}^{(k)}$ say, whereby the signal received at epoch $t$ is

$$
\mathbf{Y}_{t}=\mathbf{S X}_{t}+\mathbf{N}_{t}, \quad t=1, \ldots, T
$$

In (1) $\mathbf{S}=\left[\sqrt{\rho_{1}} \mathbf{S}^{(1)}, \ldots, \sqrt{\rho_{k}} \mathbf{S}^{(K)}\right]$ is the channel "state" matrix, $\rho_{k}$ is the $k$-th user instantaneous signal-to-noise ratio (SNR), while $\mathbf{X}_{t}=\left[X_{t}^{(1)}, \ldots, X_{t}^{(K)}\right]^{T}$ is a vector of ternary 
random variables (the "input" symbols), defined as:

$$
X_{t}^{(k)}= \begin{cases}0, & \text { if user } k \text { is idle, } \\ b_{t}^{(k)} \in\{-1,+1\}, & \text { if user } k \text { is active. }\end{cases}
$$

where $b_{t}^{(k)}$ represents the bit transmitted at epoch $t$. As to $\mathbf{N}$, it represents the additive white Gaussian noise, i.e. $\mathbf{N} \sim$ $\mathcal{N}(\mathbf{0}, \mathbf{I})$.

The evolution with time of the vector $\mathbf{X}_{t}$ is ruled by a Markov model similar to that outlined in [1]. In particular, denoting with $\mu$ the probability that a given user, active at time $t-1$, survives into epoch $t$, and with $\alpha$ the probability that a user, idle at epoch $t-1$, becomes active at epoch $t$, the basic assumptions are that users may survive or die independent of each other and that the bits emitted by a given user in consecutive time epochs are independent (i.e., the sources are memoryless). Under these conditions, the sequence $\mathbf{X}_{1: T}=$ $\left\{\mathbf{X}_{t}\right\}_{t=1}^{T}$ is a Markov chain whose state space has cardinality $3^{K}$. Thus the transition density of $\left\{\mathbf{X}_{t}\right\}_{t \in \mathbb{N}}$ factorizes as the product of the transition densities of $\left\{X_{t}^{(k)}\right\}_{t \in \mathbb{N}}$, where each $\left\{X_{t}^{(k)}\right\}_{t \in \mathbb{N}}$ is a stationary and homogeneous Markov chain with state space $\mathcal{S}=\{-1,0,1\}$ and transition probability matrix

$$
[a(i, j)]_{i, j \in \mathcal{S}}=\left(\begin{array}{ccc}
\mu / 2 & 1-\mu & \mu / 2 \\
\alpha / 2 & 1-\alpha & \alpha / 2 \\
\mu / 2 & 1-\mu & \mu / 2
\end{array}\right) .
$$

Given the above ingredients, our goal here is to study, in the said dynamic environment, the multiuser efficiency of a CDMA in the large system limit, i.e. as both the number of users $K$ and the spreading factor $N$ tends to infinity while their ratio $\beta$ remains fixed and positive. Specifically, we are interested in studying $\eta$ and MMSE when joint user activity detection and bit estimation is performed. To this end, we apply the decoupling principle to a CDMA system where all of the $K$ admissible users are assumed to be transmitting at any epoch $t$ the ternary symbols defined in (2), studying the equivalent (in the large system limit) single-user scalar channel, i.e. a scalar Gaussian channel with the same input distribution and $\operatorname{SNR}(\rho)$, and an inverse noise variance equal to the multiuser efficiency $(\eta)$ [3].

The equivalent single user scalar channel is described by the following equation

$$
Y_{t}=\sqrt{\rho} X_{t}+\frac{1}{\sqrt{\eta}} N_{t}
$$

where, with a slight abuse of notation, we have dropped the user apex. The inverse noise variance $\eta$, accounting for the degradation of the channel, is interpreted as the multi-user efficiency and can be found solving the fixed point equation

$$
\eta^{-1}=1+\beta \rho K(\eta \rho),{ }^{1}
$$

where $K$ is the MMSE (which, depends on the product $\eta \rho$ ). The solution of (3) is in general found numerically and, in

\footnotetext{
${ }^{1}$ Notice that we have dropped the expectation sign that can be found in the expression in [3] since no fading is considered in the present framework.
}

the event that it has more than one solution, $\eta$ is chosen to minimize the so-called "free-energy" (see [3] for details).

The estimate of the transmitted symbol at time $T, \widehat{X}_{T}$ say, based upon the past observations $\mathbf{Y}_{1: T}=\left\{Y_{t}\right\}_{t=1}^{T}$ is chosen to be the posterior mean, i.e. $\widehat{X}_{T}=\mathrm{E}\left[X_{T} \mid \mathbf{Y}_{1: T}\right]$. We then study the multiuser efficiency as the observation time $T$ gets large. As in [3], we can regard $\widehat{X}_{T}$ as a soft-output version of the individually optimal detectorThe conditional density of the observation at time $t$ be

$$
f\left(y_{t} \mid x_{t}\right)=\sqrt{\frac{\eta}{2 \pi}} e^{-\frac{\eta}{2}\left(y_{t}-\sqrt{\rho} x_{t}\right)^{2}}, \quad \forall x_{t} \in \mathcal{S} .
$$

whereby $\left\{X_{t}, Y_{t}\right\}_{t \in \mathbb{N}}$ is a hidden Markov process (HMP) and the likelihood up to epoch $T$ is

$f\left(\mathbf{y}_{1: T}\right)=\sum_{x_{1} \in \mathcal{S}} \cdots \sum_{x_{T} \in \mathcal{S}} \pi\left(x_{1}\right) f\left(y_{1} \mid x_{1}\right) \prod_{t=2}^{T} a\left(x_{t-1}, x_{t}\right) f\left(y_{t} \mid x_{t}\right)$,

where $\pi$ is the stationary distribution $\frac{1}{1+\alpha-\mu}\left(\frac{\alpha}{2}, 1-\mu, \frac{\alpha}{2}\right)$. The estimate $\widehat{X}_{T}$ is

$$
\begin{aligned}
\widehat{X}_{T} & =\mathrm{E}\left[X_{T} \mid \mathbf{Y}_{1: T}\right]=\sum_{x \in \mathcal{S}} x \mathrm{P}\left(\left\{X_{T}=x\right\} \mid \mathbf{Y}_{1: T}\right)= \\
& =\mathrm{P}\left(\left\{X_{T}=1\right\} \mid \mathbf{Y}_{1: T}\right)-\mathrm{P}\left(\left\{X_{T}=-1\right\} \mid \mathbf{Y}_{1: T}\right)
\end{aligned}
$$

and the MMSE at epoch $T$ is $K_{T}(\eta \rho)=\mathrm{E}\left[\left(X_{T}-\widehat{X}_{T}\right)^{2}\right]$, where the expectation is taken over the joint distribution of $\left\{X_{t}, Y_{t}\right\}_{t=1}^{T}$. After some manipulations, we obtain:

$$
\begin{aligned}
K_{T}(\eta \rho) & =\mathrm{P}\left(\left\{X_{T}=1\right\}\right)+\mathrm{P}\left(\left\{X_{T}=-1\right\}\right)-\mathrm{E}\left[\widehat{X}_{T}^{2}\right] \\
& =\frac{\alpha}{1+\alpha-\mu}-\mathrm{E}\left[\widehat{X}_{T}^{2}\right] .
\end{aligned}
$$

\section{ASYMPTOTIC ANALYSIS AND BOUNDS}

The goal of our analysis is to find an asymptotic expression for the multiuser efficiency $\eta$. To this end, we first need to prove that $\mathrm{E}\left[\widehat{X}_{T}^{2}\right]$ admits a limit as $T$ grows large, and then determine its expression. As to the former point, notice that the one-sided stationary process $\left\{X_{t}, Y_{t}\right\}_{t \in \mathbb{N}}$ can be extended to a two-sided stationary process $\left\{X_{t}, Y_{t}\right\}_{t \in \mathbb{Z}}$, whence we have:

$$
\begin{aligned}
& \mathrm{E}\left[\widehat{X}_{T}^{2}\right]=\mathrm{E}\left[\left(\mathrm{P}\left(\left\{X_{T}=1\right\} \mid \mathbf{Y}_{1: T}\right)-\mathrm{P}\left(\left\{X_{T}=-1\right\} \mid \mathbf{Y}_{1: T}\right)\right)^{2}\right] \\
& =\mathrm{E}\left[\left(\mathrm{P}\left(\left\{X_{1}=1\right\} \mid \mathbf{Y}_{-T+2: 1}\right)-\mathrm{P}\left(\left\{X_{1}=-1\right\} \mid \mathbf{Y}_{-T+2: 1}\right)\right)^{2}\right] .
\end{aligned}
$$

Since $\left(\mathrm{P}\left(\left\{X_{1}=1\right\} \mid \mathbf{Y}_{-T+2: 1}\right)-\mathrm{P}\left(\left\{X_{1}=-1\right\} \mid \mathbf{Y}_{-T+2: 1}\right)\right)^{2}$ is bounded for every $T \in \mathbb{N}$, dominated convergence gives

$$
\begin{aligned}
& \lim _{T \rightarrow+\infty} \mathrm{E}\left[\widehat{X}_{T}^{2}\right]= \\
& =\mathrm{E}\left[\left(\mathrm{P}\left(\left\{X_{1}=1\right\} \mid \mathbf{Y}_{-\infty: 1}\right)-\mathrm{P}\left(\left\{X_{1}=-1\right\} \mid \mathbf{Y}_{-\infty: 1}\right)\right)^{2}\right],
\end{aligned}
$$

where, $\forall x \in \mathcal{S}$,

$$
\lim _{T \rightarrow+\infty} \mathrm{P}\left(\left\{X_{1}=x\right\} \mid \mathbf{Y}_{-T+2: 1}\right)=\mathrm{P}\left(\left\{X_{1}=x\right\} \mid \mathbf{Y}_{-\infty: 1}\right)
$$

exists almost surely form a martingale convergence theorem by Lévy (see [5]).

Since determining a closed-form expression for $K_{T}(\eta \rho)$ as $T \rightarrow \infty$ in the general case is very challenging, we first 
analyze two special of the Markov chain $\left\{X_{t}\right\}_{t \in \mathbb{N}}$ and then, from this results, we derive a lower and upper bound for the MMSE.

\section{A. Special cases}

Let us first re-cast $\widehat{X}_{T}$ as:

$$
\begin{aligned}
& \widehat{X}_{T}= \\
& =\frac{f\left(Y_{T} \mid 1\right) \mathrm{P}\left(\left\{X_{T}=1\right\} \mid \mathbf{Y}_{1: T-1}\right)-f\left(Y_{T} \mid-1\right) \mathrm{P}\left(\left\{X_{T}=-1\right\} \mid \mathbf{Y}_{1: T-1}\right)}{\sum_{x \in \mathcal{S}} f\left(Y_{T} \mid x\right) \mathrm{P}\left(\left\{X_{T}=x\right\} \mid \mathbf{Y}_{1: T-1}\right)} \\
& =\frac{f\left(Y_{T} \mid 1\right)-f\left(Y_{T} \mid-1\right)}{f\left(Y_{T} \mid 1\right)+2 \frac{1-\mathrm{P}\left(\left\{X_{T}= \pm 1\right\} \mid \mathbf{Y}_{1: T-1}\right)}{\mathrm{P}\left(\left\{X_{T}= \pm 1\right\} \mid \mathbf{Y}_{1: T-1}\right)} f\left(Y_{T} \mid 0\right)+f\left(Y_{T} \mid-1\right)} \\
& =\frac{\sinh \left(\eta \sqrt{\rho} Y_{T}\right)}{\cosh \left(\eta \sqrt{\rho} Y_{T}\right)+\frac{1-\mathrm{P}\left(\left\{X_{T}= \pm 1\right\} \mid \mathbf{Y}_{1: T-1}\right)}{\mathrm{P}\left(\left\{X_{T}= \pm 1\right\} \mid \mathbf{Y}_{1: T-1}\right)} e^{\eta \rho / 2}},
\end{aligned}
$$

where $\mathrm{P}\left(\left\{X_{T}= \pm 1\right\} \mid \mathbf{Y}_{1: T-1}\right)=1-\mathrm{P}\left(\left\{X_{T}=0\right\} \mid \mathbf{Y}_{1: T-1}\right)$.

1) $\alpha=0, \mu=1$ : This represents a static channel, i.e. the user persists indefinitely in its state (active or not). Thus, the stationary distribution is $\pi=\left(\frac{\lambda}{2}, 1-\lambda, \frac{\lambda}{2}\right)$, for any $\lambda \in[0,1]$ ( $\lambda$ represents the probability that the user is active), and the density of $\mathbf{Y}_{1: T}$ is

$$
(1-\lambda) \prod_{t=1}^{T} f\left(y_{t} \mid 0\right)+\lambda \prod_{t=1}^{T} \frac{f\left(y_{t} \mid 1\right)+f\left(y_{t} \mid-1\right)}{2}
$$

In this case $\left\{Y_{t} \mid X_{1}=0\right\}_{t \in \mathbb{N}}$ and $\left\{Y_{t} \mid X_{1}= \pm 1\right\}_{t \in \mathbb{N}}$ are two independent processes so that one has

$$
\begin{aligned}
& \frac{1-\mathrm{P}\left(\left\{X_{T}= \pm 1\right\} \mid \mathbf{Y}_{1: T-1}\right)}{\mathrm{P}\left(\left\{X_{T}= \pm 1\right\} \mid \mathbf{Y}_{1: T-1}\right)}= \\
& \quad=\frac{1-\lambda}{\lambda} \cdot \prod_{t=1}^{T-1} \frac{f\left(Y_{t} \mid 0\right)}{\left(f\left(Y_{t} \mid 1\right)+f\left(Y_{t} \mid-1\right)\right) / 2} \\
& \stackrel{T \rightarrow+\infty}{\longrightarrow} \begin{cases}+\infty, & \text { a.s. if } X_{1}=0, \\
0, & \text { a.s. if } X_{1}= \pm 1,\end{cases}
\end{aligned}
$$

and then

$$
\begin{aligned}
& \mathrm{E}\left[\left(\widehat{X}_{T}\right)^{2}\right]= \\
& \quad=\lambda \mathrm{E}\left[\left(\widehat{X}_{T}\right)^{2} \mid X_{1}= \pm 1\right]+(1-\lambda) \mathrm{E}\left[\left(\widehat{X}_{T}\right)^{2} \mid X_{1}=0\right] \\
& \quad \stackrel{T \rightarrow+\infty}{\longrightarrow} \lambda \mathrm{E}\left[\left(\frac{f\left(Y_{1} \mid 1\right)-f\left(Y_{1} \mid-1\right)}{f\left(Y_{1} \mid 1\right)+f\left(Y_{1} \mid-1\right)}\right)^{2} \mid X_{1}= \pm 1\right],
\end{aligned}
$$

This implies, after some manipulations, that the limiting expression for the MMSE is:

$$
K_{T}(\eta \rho) \stackrel{T \rightarrow+\infty}{\longrightarrow} \lambda\left(1-\int_{\mathbb{R}} \tanh (\eta \rho-y \sqrt{\eta \rho}) \frac{e^{-\frac{y^{2}}{2}}}{\sqrt{2 \pi}} d y\right),
$$

which is exactly $\lambda$ times the canonical MMSE found in [3]. This expression allow us to say that, as the observation time tends to infinity, all the uncertainty as to the user activity can be removed: if the user is active, which happens with probability $\lambda$, errors can be due to bit estimation only.
2) $\alpha=\mu=\lambda$ : In this case $\pi=\left(\frac{\lambda}{2}, 1-\lambda, \frac{\lambda}{2}\right)$ and the density of $\mathbf{Y}^{T}$ is

$f_{T}\left(\mathbf{y}^{T}\right)=\prod_{t=1}^{T}\left(\frac{\lambda}{2} f\left(y_{t} \mid-1\right)+(1-\lambda) f\left(y_{t} \mid 0\right)+\frac{\lambda}{2} f\left(y_{t} \mid 1\right)\right)$,

i.e. the HMP degenerates into an independent process. We thus have

$$
\frac{1-\mathrm{P}\left(\left\{X_{T}= \pm 1\right\} \mid \mathbf{Y}_{1: T-1}\right)}{\mathrm{P}\left(\left\{X_{T}= \pm 1\right\} \mid \mathbf{Y}_{1: T-1}\right)}=\frac{1-\mathrm{P}\left(\left\{X_{T}= \pm 1\right\}\right)}{\mathrm{P}\left(\left\{X_{T}= \pm 1\right\}\right)}=\frac{1-\lambda}{\lambda}
$$

and then, $\forall T \in \mathbb{N}$,

$$
\mathrm{E}\left[\left(\widehat{X}_{T}\right)^{2}\right]=\mathrm{E}\left[\left(\frac{f\left(Y_{1} \mid 1\right)-f\left(Y_{1} \mid-1\right)}{f\left(Y_{1} \mid 1\right)+2 \frac{1-\lambda}{\lambda} f\left(Y_{1} \mid 0\right)+f\left(Y_{1} \mid-1\right)}\right)^{2}\right] .
$$

As to the MMSE, after some manipulations, we obtain

$$
\begin{aligned}
& K_{T}(\eta \rho)= \\
& =\lambda\left(1-\int_{\mathbb{R}} \frac{\sinh (\eta \rho-y \sqrt{\eta \rho})}{\cosh (\eta \rho-y \sqrt{\eta \rho})+\frac{1-\lambda}{\lambda} e^{\eta \rho / 2}} \frac{e^{-\frac{y^{2}}{2}}}{\sqrt{2 \pi}} d y\right) .
\end{aligned}
$$

which corresponds to the MMSE of the "one-shot" estimation of user activity and data found in [4]. A case of particular interest is $\alpha=\mu=1 / 2$, which, as will be seen later, corresponds to the worst case MMSE for large values of $\rho$.

Observation 3.1: In general, the estimation of the state $X_{T}$ based upon $\mathbf{Y}_{1: T}$ corresponds to performing both user activity detection and data estimation. Since the transmitted bits are independent, past observations may only be helpful in the latter task. The two extrema are the static case $(\alpha=0, \mu=1)$, where channel occupancy estimation is improved for larger $T$, and independent observation case $(\alpha=\mu)$, where memoryless processing is optimum. In general, as $\alpha$ approaches zero and $\mu$ approaches 1 (which means that the user persists being idle or active for long time) larger values of $T$ result in larger gains.

\section{B. Upper and lower bound}

Given the form of the stationary distribution, we have that $\mathrm{P}\left(\left\{X_{1}= \pm 1\right\}\right)=\frac{\alpha}{1+\alpha-\mu}$. The best case would be clearly the static case with initial distribution $\left(\frac{\lambda}{2}, 1-\lambda, \frac{\lambda}{2}\right)$ and $\lambda=$ $\frac{\alpha}{1+\alpha-\mu}$ since, when $T \rightarrow+\infty$, the uncertainty as to the user activity is completely removed and data detection only need to be performed. A lower bound for the MMSE, then, is that in equation (4). Developing (4) we have

$$
\begin{aligned}
1-\int_{\mathbb{R}} \tanh ( & \eta \rho-y \sqrt{\eta \rho}) \frac{e^{-y^{2} / 2}}{\sqrt{2 \pi}} d y= \\
= & \int_{\mathbb{R}} \frac{2 e^{\sqrt{\eta \rho}(y-\sqrt{\eta \rho})}}{e^{\sqrt{\eta \rho}(y-\sqrt{\eta \rho})}+e^{-\sqrt{\eta \rho}(y-\sqrt{\eta \rho})}} \frac{e^{-y^{2} / 2}}{\sqrt{2 \pi}} d y= \\
= & 2 e^{-\eta \rho / 2} \int_{\mathbb{R}} \frac{1}{e^{\sqrt{\eta \rho} y}+e^{-\sqrt{\eta \rho} y}} \frac{e^{-y^{2} / 2}}{\sqrt{2 \pi}} d y= \\
= & 2 \int_{-\infty}^{0} \frac{1}{1+e^{2 y \sqrt{\eta \rho} y}} \frac{e^{-\frac{(y-\sqrt{\eta \rho})^{2}}{2}}}{\sqrt{2 \pi}} d y+ \\
& +2 \int_{0}^{+\infty} \frac{1}{1+e^{-2 y \sqrt{\eta \rho} y}} \frac{e^{-\frac{(y+\sqrt{\eta \rho})^{2}}{2}}}{\sqrt{2 \pi}} d y=
\end{aligned}
$$




$$
\begin{aligned}
= & 2 \sum_{k=0}^{+\infty}(-1)^{k}\left(\int_{-\infty}^{0} e^{2 k \sqrt{\eta \rho} y} \frac{e^{-\frac{(y-\sqrt{\eta \rho})^{2}}{2}}}{\sqrt{2 \pi}} d y+\right. \\
& \left.+\int_{-\infty}^{0} e^{-2 k \sqrt{\eta \rho} y} \frac{e^{-\frac{(y+\sqrt{\eta \rho})^{2}}{2}}}{\sqrt{2 \pi}} d y\right)= \\
= & 4 \sum_{k=0}^{+\infty}(-1)^{k} e^{\frac{(2 k+1)^{2} \eta \rho}{2}} Q\left(\sqrt{(2 k+1)^{2} \eta \rho}\right) \approx \\
\approx & \frac{4 e^{-\eta \rho / 2}}{\sqrt{2 \pi \eta \rho}} \sum_{k=0}^{+\infty} \frac{(-1)^{k}}{2 k+1}=\sqrt{\frac{\pi}{2 \eta \rho e^{\eta \rho}}} .
\end{aligned}
$$

Notice that in the above chain of equalities, no approximation has been advocated except that in the last step, where the $Q$ function has been replaced with its asymptotic behavior for large $\rho$. Thus all but the last equalities hold for any value of $\rho$, and are consistent with those obtained in [6] for large $\rho$. We thus obtain

$$
K_{T}(\eta \rho) \geq \lambda \sqrt{\frac{\pi}{2 \eta \rho}} e^{-\eta \rho / 2},
$$

with equality if the channel is static.

As concerns the upper bound, let $\gamma=\mathrm{P}\left(\left\{X_{T}= \pm 1\right\} \mid\right.$ $\left.\mathbf{Y}_{1: T-1}\right)$. Then, the MMSE at epoch $T$ conditioned upon the past observations $\mathbf{Y}_{1: T-1}$ can be also written as

$$
\begin{aligned}
& K_{T}\left(\eta \rho \mid \mathbf{Y}_{1: T-1}\right)=\gamma-\int_{\mathbb{R}} \widehat{X}^{2}\left(\frac{\gamma}{2} f(y \mid 1)+(1-\gamma) f(y \mid 0)+\right. \\
& \left.+\frac{\gamma}{2} f(y \mid-1)\right) d y= \\
& =\int_{\mathbb{R}} \frac{\gamma \frac{f(y \mid)+f(y \mid-1)}{2}(1-\gamma) f(y \mid 0)+\gamma^{2} f(y \mid 1) f(y \mid-1)}{\frac{\gamma}{2} f(y \mid 1)+(1-\gamma) f(y \mid 0)+\frac{\gamma}{2} f(y \mid-1)} d y,
\end{aligned}
$$

so that $K_{T}(\eta \rho)=\mathrm{E}\left[K_{T}\left(\eta \rho \mid \mathbf{Y}_{1: T-1}\right)\right]$. The second derivative of $K_{T}\left(\eta \rho \mid \mathbf{Y}_{1: T-1}\right)$ with respect to $\gamma$ is

$$
-\frac{1}{2} \int_{\mathbb{R}} \frac{f^{2}(y \mid 0)(f(y \mid 1)-f(y \mid-1))^{2}}{\left(\frac{\gamma}{2} f(y \mid 1)+(1-\gamma) f(y \mid 0)+\frac{\gamma}{2} f(y \mid-1)\right)} d y,
$$

showing that the MMSE is a concave function of $\gamma$ and attains an absolute maximum for $\gamma \in[0,1]$. Furthermore, equation (5) can be also written as

$$
\begin{aligned}
& K_{T}\left(\eta \rho \mid \mathbf{Y}_{1: T-1}\right)= \\
& =\int_{\mathbb{R}} \frac{\frac{\gamma}{2}(1-\gamma) f(y \mid 0)+\frac{\gamma^{2}}{2} f(y \mid-1)}{\frac{\gamma}{2} f(y \mid 1)+(1-\gamma) f(y \mid 0)+\frac{\gamma}{2} f(y \mid-1)} f(y \mid 1) d y+ \\
& +\int_{\mathbb{R}} \frac{\frac{\gamma}{2}(1-\gamma) f(y \mid 0)+\frac{\gamma^{2}}{2} f(y \mid-1)}{\frac{\gamma}{2} f(y \mid 1)+(1-\gamma) f(y \mid 0)+\frac{\gamma}{2} f(y \mid-1)} f(y \mid-1) d y .
\end{aligned}
$$

Divide now the interval $[0,1]$ into two sub-intervals, $[0,3 / 4]$ and $[3 / 4,1]$. For large $\rho$ and $\gamma \in[0,3 / 4]$, we have

$$
\begin{aligned}
& K_{T}\left(\eta \rho \mid \mathbf{Y}_{1: T-1}\right) \approx \int_{\mathbb{R}} \frac{\frac{\gamma}{2} f(y \mid 1)(1-\gamma) f(y \mid 0)}{\frac{\gamma}{2} f(y \mid 1)+(1-\gamma) f(y \mid 0)} d y+ \\
& +\int_{\mathbb{R}} \frac{\frac{\gamma}{2} f(y \mid-1)(1-\gamma) f(y \mid 0)}{(1-\gamma) f(y \mid 0)+\frac{\gamma}{2} f(y \mid-1)} d y=
\end{aligned}
$$

$$
\begin{aligned}
& =2 \int_{\mathbb{R}} \frac{\frac{\gamma}{2} f(y \mid 1)(1-\gamma) f(y \mid 0)}{\frac{\gamma}{2} f(y \mid 1)+(1-\gamma) f(y \mid 0)} d y= \\
& =2 \int_{\mathbb{R}} \frac{\gamma(1-\gamma) e^{\sqrt{\eta \rho}\left(y-\frac{\sqrt{\eta \rho}}{2}-\frac{\ln 2}{\sqrt{\eta \rho}}\right)}}{\gamma e^{\sqrt{\eta \rho}\left(y-\frac{\sqrt{\eta \rho}}{2}-\frac{\ln 2}{\sqrt{\eta \rho}}\right)}+(1-\gamma)} \frac{e^{-y^{2} / 2}}{\sqrt{2 \pi}} d y= \\
& =2 \int_{\mathbb{R}} \frac{\gamma(1-\gamma) e^{\frac{\sqrt{\eta \rho}}{2}\left(y-\frac{\sqrt{\eta \rho}}{2}-\frac{\ln 2}{\sqrt{\eta \rho}}\right)} \frac{e^{-y^{2} / 2}}{\sqrt{2 \pi}}}{2\left(y-\frac{\sqrt{\eta \rho}}{2}-\frac{\ln 2}{\sqrt{\eta \rho}}\right)}+(1-\gamma) e^{-\frac{\sqrt{\eta \rho}}{2}\left(y-\frac{\sqrt{\eta \rho}}{2}-\frac{\ln 2}{\sqrt{\eta \rho}}\right)} d y= \\
& =2 \int_{\mathbb{R}} \frac{\gamma(1-\gamma) e^{\frac{\sqrt{\eta \rho}}{2}} y}{\gamma e^{\frac{\sqrt{\eta \rho}}{2}} y+(1-\gamma) e^{-\frac{\sqrt{\eta \rho}}{2}} y} \frac{e^{-\left(y+\frac{\sqrt{\eta \rho}}{2}+\frac{\ln 2}{\sqrt{\eta \rho}}\right)^{2} / 2}}{\sqrt{2 \pi}} d y \approx \\
& \approx 2 \int_{\mathbb{R}} \frac{\gamma(1-\gamma) e^{\frac{\sqrt{\eta \rho}}{2} y}}{\gamma e^{\frac{\sqrt{\eta \rho}}{2} y}+(1-\gamma) e^{-\frac{\sqrt{\eta \rho}}{2}} y} \frac{e^{-\left(y+\frac{\sqrt{\eta \rho}}{2}\right)^{2} / 2}}{\sqrt{2 \pi}} d y= \\
& =2 \int_{\mathbb{R}} \frac{\gamma f\left(y \mid \frac{1}{2}\right)(1-\gamma) f\left(y \mid-\frac{1}{2}\right)}{\gamma f\left(y \mid \frac{1}{2}\right)+(1-\gamma) f\left(y \mid-\frac{1}{2}\right)} d y,
\end{aligned}
$$

Notice that the above approximation for large $\rho$ is still concave in $\gamma$ as its second derivative is

$$
-2 \int_{\mathbb{R}} \frac{\left(f\left(y \mid \frac{1}{2}\right) f\left(y \mid-\frac{1}{2}\right)\right)^{2}}{\left(\gamma f\left(y \mid \frac{1}{2}\right)+(1-\gamma) f\left(y \mid-\frac{1}{2}\right)\right)^{3}} d y,
$$

Since $K_{T}\left(\eta \rho \mid \mathbf{Y}_{1: T-1}\right)$ is itself concave, its symmetry for large $\rho$ implies that the maximum cannot be attained for $\gamma \in[3 / 4,1]$, whereby, for large $\rho$, the MMSE is maximum at $\gamma=1 / 2$ and we have

$$
\begin{aligned}
& K_{T}\left(\eta \rho \mid \mathbf{Y}_{1: T-1}\right) \leq \int_{\mathbb{R}} \frac{e^{\frac{\sqrt{\eta \rho}}{2}} y}{e^{\frac{\sqrt{\eta \rho}}{2}} y+e^{-\frac{\sqrt{\eta \rho}}{2}} y} \frac{e^{-\left(y+\frac{\sqrt{\eta \rho}}{2}\right)^{2} / 2}}{\sqrt{2 \pi}} d y= \\
& =\frac{1}{2} \int_{\mathbb{R}} \frac{2 e^{\sqrt{\frac{\eta \rho}{4}}\left(y-\sqrt{\frac{\eta \rho}{4}}\right)}}{e^{\sqrt{\frac{\eta \rho}{4}}\left(y-\sqrt{\frac{\eta \rho}{4}}\right)}+e^{-\sqrt{\frac{\eta \rho}{4}}}\left(y-\sqrt{\frac{\eta \rho}{4}}\right)} \frac{e^{-y^{2} / 2}}{\sqrt{2 \pi}} d y,
\end{aligned}
$$

This is exactly the same expression (with $\rho / 4$ instead of $\rho$ ) derived for the lower bound and, then, $K_{T}\left(\eta \rho \mid \mathbf{Y}_{1: T-1}\right) \leq$ $\sqrt{\frac{\pi}{2 \eta \rho}} e^{-\eta \rho / 4}$, for any $\mathbf{Y}_{1: T-1}$, i.e.

$$
K_{T}(\eta \rho) \leq \sqrt{\frac{\pi}{2 \eta \rho}} e^{-\eta \rho / 8}
$$

with equality if $\gamma=\mathrm{P}\left(\left\{X_{T}= \pm 1\right\} \mid \mathbf{Y}_{1: T-1}\right)=1 / 2$, i.e. if $\alpha=\mu=1 / 2$ (independent case). This can be interpreted as follows. For large SNR's data detection errors can be neglected (since the distance between -1 and +1 is twice the distance between 0 and \pm 1 ) and errors are primarily due to user activity estimation (which amounts to discriminating between \pm 1 and 0 ): the worst case, then, is when \pm 1 and 0 are equiprobable.

Summing up, the smallest MMSE is obtained in the stationary case with initial distribution $\left(\frac{\lambda}{2}, 1-\lambda, \frac{\lambda}{2}\right)$ and, for large SNR's, the worst-case MMSE corresponds to the memoryless channel $\alpha=\mu=1 / 2$, i.e.:

$$
\lambda \sqrt{\frac{\pi}{2 \eta \rho}} e^{-\eta \rho / 2} \leq K_{T}(\eta \rho) \leq \sqrt{\frac{\pi}{2 \eta \rho}} e^{-\eta \rho / 8} .
$$

The limiting values of $\eta$ can be found though equation (3) and the above limiting value of the MMSE. 


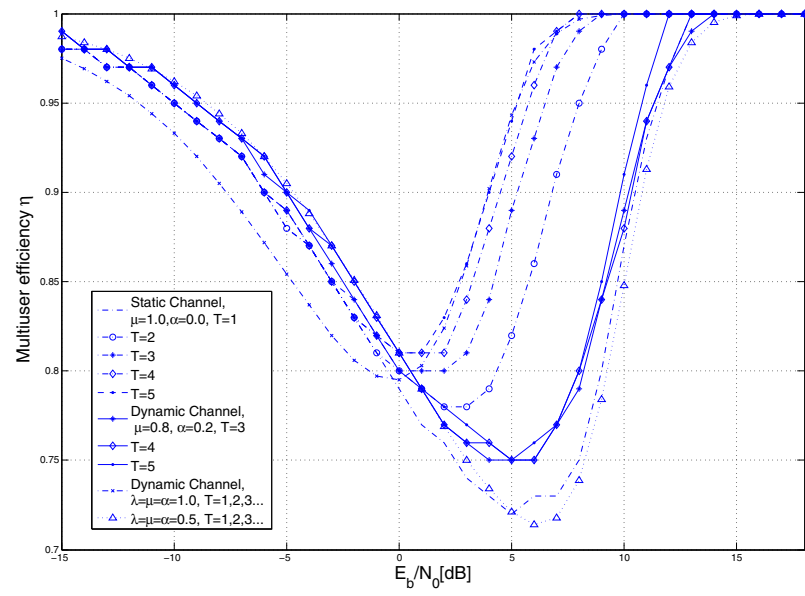

Fig. 1. Multiuser efficiency versus the SNR for different dynamics: static channel, independent case and $(\alpha, \mu)=(0.2,0.8)$ case.

\section{NUMERICAL RESULTS}

The analytical results found in the previous sections are now validated through numerical Monte Carlo simulations. A system load of $\beta=3 / 7$ has been used and the observation time $T$ has been varied between 1 and 5 , which, given the small cardinality of the state space $\mathcal{S}$, represents a good approximation for the asymptotic analysis.

Figs. 1 shows the multiuser versus the SNR. In both figures a typical dynamic case, $(\alpha, \mu)=(0.2,0.8)$, is compared with the static case $(\alpha, \mu)=(0,1)$ and with the independent case $(\alpha, \mu)=\left(\frac{1}{2}, \frac{1}{2}\right)$. As expected, in the static case, the performances improves as the observation time increases while, in the independent case, past observations do not increases estimation accuracy. In the typical $(0.2,0.8)$ dynamic case we can see that the performance improvement quickly saturate with $T$, given the limited "memory" of the Markov chain $\left\{X_{t}\right\}$. Figs. 2 and 3 focus on the independent case. The former shows the multiuser efficiency versus the SNR for different values of $\lambda=\mu=\alpha$ while in the latter the MMSE is reported versus $\lambda$ for different SNR's. It can be seen that, as the SNR increases, the worst-case MMSE (the $\times$ markers) moves towards $\lambda=1 / 2$, this further confirming the correctness of the upper bound derived in Section III-B.

\section{Conclusions}

This paper provides a large-system analysis of a dynamic multiuser system where the activity of users may vary over time following a Markovian model. Exploiting the largesystem equivalent scalar channel, the MMSE and the multiuser efficiency has been found for the case of joint user activity detection and bit estimation based on present and past observations. An asymptotic analysis, as the observation time gets large, has been given, along with bounds on the MMSE which have shown that the best-case MMSE is obtained in the stationary case and, for high SNR's, the worst-case MMSE for the independent case with $\alpha=\mu=1 / 2$.

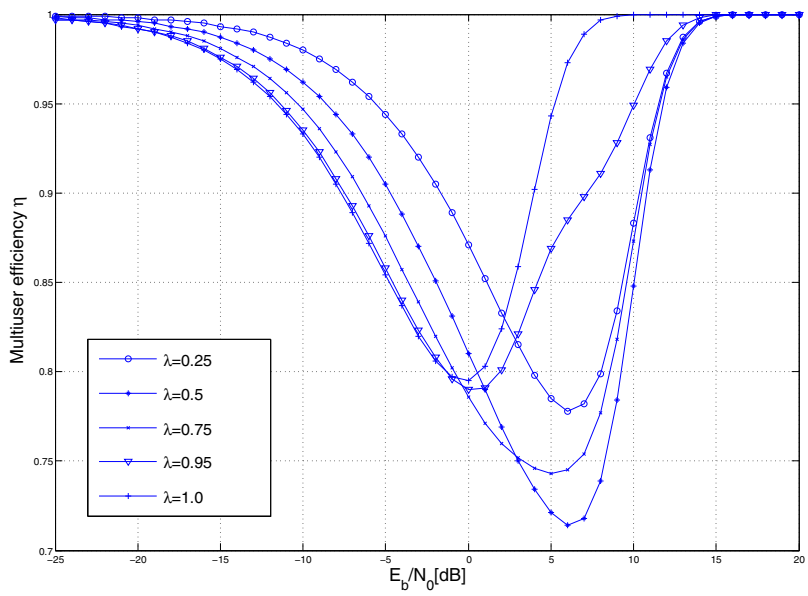

Fig. 2. Multiuser efficiency versus the SNR for the independent processes and different values of $\mu=\alpha=\lambda$.

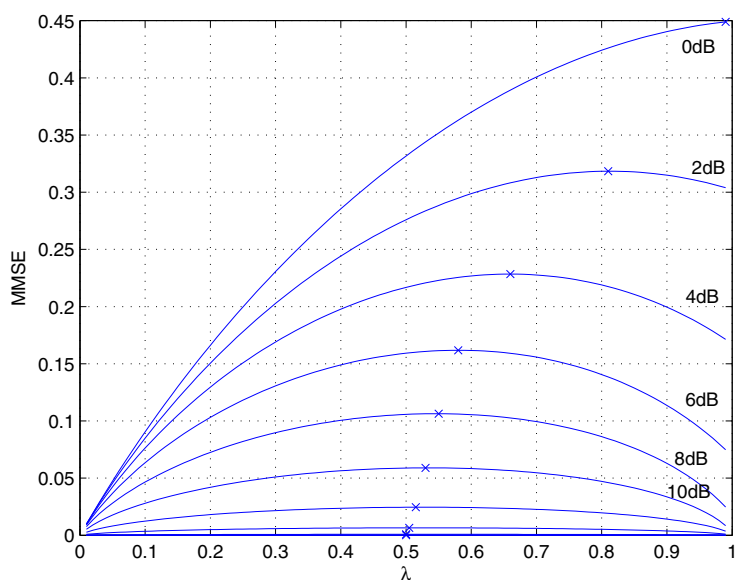

Fig. 3. MMSE versus the SNR for the independent processes and different values of $\mu=\alpha=\lambda$.

\section{ACKNOWLEDGEMENTS}

This work was supported by the Spanish Ministry of Education and Science under Project TEC2006- 01428/TCM, and by the STREP project No. IST026905 (MASCOT) within the 6th framework program of the European Commission.

\section{REFERENCES}

[1] E. Biglieri and M. Lops, "Multiuser detection in a dynamic environmentPart I: User identification and data detection," IEEE Trans. Inform. Theory, vol. 53, no. 9, pp. 3158-3170, September 2007.

[2] D. Angelosante, E. Biglieri, and M. Lops, "Multiuser detection in a dynamic environment: Joint user identification and parameter estimation," in Proc. IEEE Int. Symp. on Information Theory, Nice, France, June 2007.

[3] D. Guo and S. Verdù, "Randomly spread CDMA: Asymptotics via statistical physics," IEEE Trans. Inform. Theory, vol. 51, no. 6, pp. 19832007, June 2005.

[4] A. Tauste Campo and E. Biglieri, "Large-system analysis of static multiuser detection with an unknown number of users," in Proc. of IEEE Int. Work. on Computational Advances in Multi-Sensor Adaptive Processing (CAMSAP'07), Saint Thomas, U.S., December 2007.

[5] A. N. Shiryaev, Probability. New York: Springer, 1995.

[6] A. Lozano, A. M. Tulino, and S. Verdù, "Optimum power allocation for parallel gaussian channels with arbitrary input distributions," IEEE Trans. Inform. Theory, vol. 52, no. 7, pp. 3033-3051, July 2006. 